\title{
Chest wall thickness may limit adequate drainage of tension pneumothorax by needle thoracocentesis
}

\author{
Simon Britten, Simon H Palmer
}

\begin{abstract}
Tension pneumothorax in a large man was inadequately drained by needle thoracocentesis with a $4.5 \mathrm{~cm}$ cannula. Unsuccessful needle thoracocentesis of a clinical tension pneumothorax in a large patient should be followed immediately by chest drain insertion, without local anaesthetic, as dictated by clinical urgency. If the clinical situation is still not improved other diagnoses should be considered. (F Accid Emerg Med 1996;13:426-427)
\end{abstract}

Key terms: tension pneumothorax; needle thoracocentesis

In order to perform needle thoracocentesis for life threatening tension pneumothorax the advanced trauma life support guidelines recommend use of a cannula 3 to $6 \mathrm{~cm}$ long inserted into the second intercostal space in the midclavicular line. ${ }^{1}$ We present a case of tension pneumothorax which was inadequately drained by this technique.

\section{Case report}

A 52 year old builder sustained blunt injury to the right posterior chest when hit by a $6 \mathrm{~m}$ sheet of corrugated roofing, which had fallen 8 $\mathrm{m}$. In the ambulance he was in extremis, conscious but unable to speak and cyanosed with a respiratory rate greater than $40 / \mathrm{min}$. There was no chest wall movement despite great ventilatory effort. Surgical emphysema of the abdominal and chest walls and the subcutaneous tissues of head and neck was present.

Right rib fractures were evident. The clinical

Royal United Hospital, Bath, United Kingdom S Britten

S H Palmer

Correspondence to: Mr S Britten FRCS, Department of Orthopaedics, Royal United Hospital, Bath BA1 3NG.

Accepted for publication 14 May 1996 room where the clinical signs of tension pneumothorax recurred. A chest drain was inserted and a large volume of air under pressure drained immediately, leading to rapid improvement in chest wall movement and oxygen saturation measured by a pulse oximeter. Subsequent chest $x$ ray revealed fractures of right posterior ribs 2 to 6 , right lung contusion, gross subcutaneous emphysema, mediastinal emphysema, and a residual right pneumothorax. The patient recovered uneventfully and was discharged on day 11 .

\section{Discussion}

The tension pneumothorax was successfully decompressed in the ambulance but recurred during the brief transfer to the resuscitation room despite the cannula being left in situ. We suggest that the chest wall thickness of this large patient prevented adequate pleural drainage by needle thoracocentesis. The tip of the $4.5 \mathrm{~cm}$ cannula could reach the pleural cavity only while pressure was applied to insert the cannula, causing partial compression of the chest wall (figure, panel A). However, once the cannula was left in situ, the chest wall would reform, causing the cannula to back out. This would leave its tip short of the pleural cavity (figure, panel B), leading to loss of pleural drainage and recurrence of tension pneumothorax.

While needle thoracocentesis is a useful technique for the decompression of life threatening tension pneumothorax, we suggest that difficulty in achieving successful decompression may be encountered where a patient is very muscular and in the presence of gross subcutaneous emphysema. In such a case, the chest wall thickness is greater than the length of the cannula.

Therefore we recommend that unsuccessful needle thoracocentesis of a clinical tension pneumothorax in a large patient should be followed immediately by chest drain insertion, without local anaesthetic, as dictated by clinical urgency. Alternatively, a longer cannula may succeed as a temporising measure where a $4.5 \mathrm{~cm}$ cannula has failed. If the clinical situation is still not improved other diagnoses should be considered.

Such difficulties with needle thoracocentesis may also be of relevance to ambulance services as they develop protocols governing the 


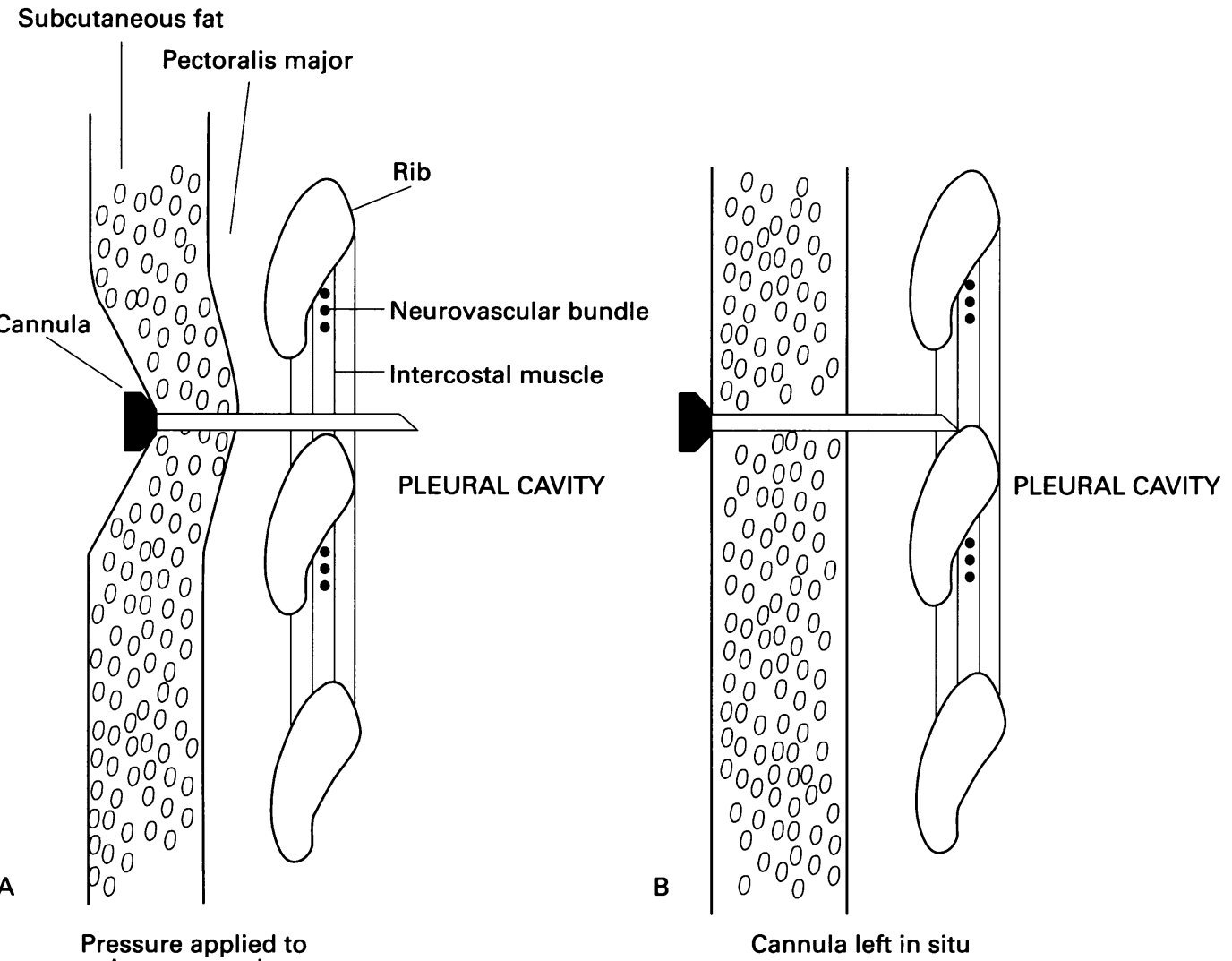

Relation between chest wall and cannula.

treatment of tension pneumothorax by paramedic staff.
Department of Accident and Emergency, Bristol Royal Infirmary, Bristol BS2 8HW, United Kingdom R Malhotra G Hughes

Correspondence to: Dr Raman Malhotra.

\title{
Methaemoglobinaemia presenting with status epilepticus
}

\author{
Raman Malhotra, Geoff Hughes
}

\begin{abstract}
A case is reported of methaemoglobinaemia presenting with recurrent fits in the absence of cyanosis. A low oxygen saturation measured on pulse oximetry that fails to improve with oxygen treatment, the presence of "chocolate brown" blood that does not change on exposure to air, and a high $\mathrm{PaO}_{2}$ arterial blood gas with oxygen therapy should support such a diagnosis. A diagnostic blood methaemoglobin level should be obtained.

$(\Im$ Accid Emerg Med 1996;13:427-430)
\end{abstract}

Key terms: amyl nitrite; cyanosis; convulsions; methaemoglobinaemia

Fitting is frequently seen in accident and emergency ( $A \& E)$; common causes include hypoglycaemia, epilepsy, alcohol withdrawal or excess, infection (including meningitis, en cephalitis, or childhood febrile fits), head injury, hypoxia, and intracranial pathology. ${ }^{1}$

Methaemoglobinaemia is usually described as an uncommon cause of cyanosis in patients presenting to the A\&E department, ${ }^{2-5}$ but has also been reported to cause chest pain ${ }^{46}$ or coma ${ }^{6-8}$ We report a case of methaemoglobinaemia presenting with recurrent fits.

\section{Case report}

A 31 year old male was brought to the $A \& E$ department by ambulance after being found on a pavement having repeated convulsions. The fits were described as recurrent, lasting approximately two minutes each with threeminute intervals, during which time he was unresponsive to voice or pain. He was unaccompanied and no other history was available.

On arrival he was in status epilepticus. Only the upper half of his body was convulsing, with 